ISSN: 1782-2041

DOI: $10.25518 / 1782-2041.1126$

\title{
Bibliographie de Robert Brisart (1953-2015)
}

Par DENIS SERON

Université de Liège, FNRS

[1] Brisart, R. (1978\a). Recension de: M. Henry, Marx, vol. 1: Une philosophie de la réalité ; vol. 2 : Une philosophie de l'économie. Revue philosophique de Louvain 76, 32, p. 481-485.

[2] Brisart, R. (1978lb). Recension de : W.W. Fuchs, Phenomenology and the Metaphysics of Presence: An Essay in the Philosophy of Edmund Husserl. Revue philosophique de Louvain 76, 32, p. 493-496.

[3] Brisart, R. (1979). Recension de : T.A. Fay, Heidegger : The Critique of Logic. Revue philosophique de Louvain 77, 36, p. 594-598.

[4] Brisart, R. (1980). La possibilisation du temps et la temporalisation du possible [étude critique]. Revue philosophique de Louvain 78, 37, p. 99 117.

[5] Brisart, R. (1981\a). Introduction. Cahiers du Centre d'études phénoménologiques 1, «Autour de l'être, du temps et de l'autre », p. 3-6.

[6] Brisart, R. (1981\b). Temps et affectivité. Cahiers du Centre d'études phénoménologiques 1, "Autour de l'être, du temps et de l'autre», p. 21-43.

[7] Brisart, R. $(1981 \backslash c)$. Présence et Être : Étude sur l'approfondissement de la phénoménologie dans les "Marburger Vorlesungen» de Heidegger. Revue philosophique de Louvain 79, 41, p. 28-70.

[8] Brisart, R. (1982\a). L'intentionnalité comme «Titre d'un problème central » selon Heidegger. Cahiers du Centre d'études phénoménologiques 2, « Sur la temporalité, l'intentionnalité, l'imaginaire», p. 3384.

[9] Brisart, R. (1982\b). Phénoménologie et historicité selon Jan Patočka [étude critique]. Revue philosophique de Louvain 80, 48, p. 669-681. 
[10] Brisart, R. (1983\a). Du sens phénoménologique de la mémoire à notre époque. Cahiers du Centre d'études phénoménologiques 3, « Raison et finitude », p. 33-84.

[11] Brisart, R. (1983\b). Recension de : E. Husserl, Recherches phénoménologiques pour la constitution, trad. É. Escoubas. Revue philosophique de Louvain 81, 52, p. 652-658.

[12] Brisart, R. (1986). Existence et culpabilité dans Sein und Zeit. In : P. Jonckheere (éd.), Phénoménologie et analyse existentielle. Louvainla-Neuve : Presses universitaires de Louvain, p. 71-93; $2^{\mathrm{e}}$ éd. (1989), Bruxelles : De Boeck Université, p. 63-81.

[13] Brisart, R. (1988\a). Remarques sur la conception de la réduction phénoménologique chez Heidegger. In : G. Florival (éd.), Figures de la finitude: Études d'anthropologie philosophique III. Louvain: Bibliothèque philosophique de Louvain, p. 33-52.

[14] Brisart, R. (1988\b). La métaphysique de Heidegger. In : S. Ijsseling \& J. Taminiaux (éds.), Heidegger et l'idée de la phénoménologie. Dordrecht : Kluwer, p. 217-238.

[15] Brisart, R. (1988\c). Avant-propos. Études phénoménologiques 8, p. 37.

[16] Brisart, R. (éd.) (1988\d). Études phénoménologiques 8, numéro « Hans Jonas ».

[17] Jonas, H. (1988). La science comme expérience vécue, trad. R. Brisart. In [16], p. 9-32. [H. Jonas (1987). Wissenschaft als persönliches Erlebnis. In: H. Jonas, Wissenschaft als persönliches Erlebnis. Göttingen : Vandenhoeck \& Ruprecht, p. 7-31.]

[18] Brisart, R. (1990). Nature et liberté dans l'ontologie fondamentale de Heidegger: De la radicalisation d'une antinomie moderne à l'acosmisme existential. Revue philosophique de Louvain 88, 80, p. 524-552.

[19] Brisart, R. (1991). La phénoménologie de Marbourg ou la résurgence métaphysique chez Heidegger à l'époque de Sein und Zeit. Bruxelles : Publications des Facultés universitaires Saint-Louis.

[20] Brisart, R. (1993\a). La réduction et l'irréductible phénoménologiques : Husserl critique de Heidegger. In [22], p. 139-185.

[21] Brisart, R. (1993lb). Préface. In : L. Van Eynde, L'ontologie acosmique: La crise de la modernité chez Pascal et Heidegger. Bruxelles : Publications des Facultés universitaires Saint-Louis, p. 3-9.

[22] Brisart, R. \& Célis, R. (éds.) (1993\a). L'évidence du monde : Méthode et empirie de la phénoménologie. Bruxelles : Publications des Facultés universitaires Saint-Louis. 
[23] Brisart, R. \& Célis, R. (1993\b). Avant-propos. In : L'évidence du monde : Méthode et empirie de la phénoménologie. Bruxelles : Publications des Facultés universitaires Saint-Louis, p. 7-8.

[24] Brisart, R. (1994). Avant-propos. Recherches husserliennes 1, p. 3-14.

[25] Brisart, R. (1995). Référence et traduisibilité chez Husserl : Approche phénoménologique de la commensurabilité du sens. In [26], p. 393-451.

[26] Brisart, R. \& Célis, R. (éds.) (1995\a). La voix des phénomènes : Contributions à une phénoménologie du sens et des affects. Bruxelles : Publications des Facultés universitaires Saint-Louis.

[27] Brisart, R. \& Célis, R. (1995\b). Avant-propos. In [26], p. 7-10.

[28] Brisart, R. (1998\a). Le tournant logique de Husserl en 1891: La recension de Schröder et ses antécédents. Recherches husserliennes 10, p. 3-34.

[29] Brisart, R. (1998\b). La découverte des processus signitifs dans la première œuvre mathématique de Husserl (1887-1891). In [30], p. 9 62.

[30] Benoist, J., Brisart, R., English, J. (éds.) (1998\a). Liminaires phénoménologiques : Le développement de la théorie de la signification chez Husserl. Bruxelles: Publications des Facultés universitaires SaintLouis, p. 9-62.

[31] Benoist, J. , Brisart, R., English, J. (1998\b). Préface. In [30], p. 7.

[32] Natorp, P. (2000). Sur la question de la méthode logique en rapport aux Prolégomènes à la logique pure d'Ed. Husserl, trad. R. Brisart. Recherches husserliennes 13, p. 3-18. [P. Natorp (1901). Zur Frage der logischen Methode, mit Beziehung auf Husserls Prolegomena zur reinen Logik. Kant-Studien 6, p. 270-283.]

[33] Brisart, R. (2001). Retour à Husserl. Magazine littéraire 11, 403, p. 36.

[34] Brisart, R. (2002\a). Husserl et Bolzano: Le lien sémantique. Recherches husserliennes 18, p. 3-29.

[35] Brisart, R. (2002ไb). Avant-propos. In [36], p. 7-11.

[36] Brisart, R. (éd.) (2002\c). Husserl et Frege: Les ambiguïtés de l'antipsychologisme. Paris : Vrin.

[37] Brisart, R. (2002\d). Le problème de l'abstraction en mathématique : l'écart initial de Husserl par rapport à Frege entre 1891 et 1894. In [36], p. 13-47.

[38] Brisart, R. (2002le). La logique de Husserl en 1900 à l'épreuve du néokantisme marbourgeois: La recension de Natorp. Phänomenologische Forschungen, p. 183-204.

[39] Brisart, R. (2002\f). La vérité est ailleurs. Droit et société 2-3, 51-52, p. $423-432$. 
[40] Brisart, R. (2003\a). Le général et l'abstrait: Sur la maturation des Recherches logiques de Husserl. In : D. Fisette \& S. Lapointe (éds.), Aux origines de la phénoménologie: Husserl et le contexte des Recherches logiques. Paris : Vrin ; Sainte-Foy : Presses de l'Université Laval, p. 21-40.

[41] Brisart, R. (2003\b). Das Unreduzierbare in phänomenologischer Hinsicht, trad. par K. Schulz de [20] fortement modifié. In : R. Kühn \& M. Staudigl (éds.), Epoché und Reduktion: Formen und Praxis der Reduktion in der Phänomenologie. Würzburg: Königshausen \& Neumann, p. 81-106.

[42] Brisart, R. (2003 \c). La théorie de l'objet dans les Recherches logiques de Husserl. In: E. Husserl, La représentation vide suivi de Les Recherches logiques, une œuvre de percée, éd. J. Benoist \& J.F. Courtine. Paris : Presses Universitaires de France, p. 125-139.

[43] Brisart, R. (2006). Il recupero heideggeriano dell'onto-teologia a Marburgo e la questione della fenomenologia, trad. P. Amato. In : E. Mazzarella (éd.), Heidegger a Marburgo (1923-1928). Genova : Il Melangolo, p. 55-72.

[44] Brisart, R. (2007\a). Les premières articulations du fonctionnement intentionnel: Le projet d'un Raumbuch chez Husserl entre 1892 et 1894. Philosophiques 34, 2, p. 259-272.

[45] Brisart, R. (2007\b). L'idéalisme dans la proto-phénoménologie. In [46], p. 13-24.

[46] Brisart, R. \& Maesschalck, M. (éds.) (2007\a). Idéalisme et phénoménologie. Hildesheim Zürich New York : Georg Olms.

[47] Brisart, R. \& Maesschalck, M. (2007\b). Avant-propos. In [46], p. 5-9.

[48] Brisart, R. (2009\a). Perception, sens et vérité : La phénoménologie à l'épreuve de l'opacité référentielle. Topos 2-3 (22), p. 33-47.

[49] Brisart, R. (2009 \b). La théorie des assomptions chez le jeune Husserl. Philosophiques 36, 2, p. 399-425.

[50] Brisart, R. (2011\a). Husserl et la no ready-made theory: la phénoménologie dans la tradition constructiviste. Bulletin d'analyse phénoménologique 7, 1, p. 3-36 (série Actes, 4).

[51] Brisart, R. $(2011 \backslash b)$. Husserl et le mythe des objets. Philosophie 111, p. $26-51$.

[52] Brisart, R. (2011 \c). Husserl et l'affaire des démonstratifs : À propos de la référence en régime noématique. Revue philosophique de Louvain 109,2 , p. 245-269.

[53] Brisart, R. (2012). True objects and fulfilments under assumption in the young Husserl. Axiomathes 22, p. 75-89. 
[54] Brisart, R. (2013). L'expérience perceptive et son passif : À propos des sensations dans le constructivisme de Husserl. Philosophie 119, p. 3363.

[55] Brisart, R. (2015). Objects as posits from a phenomenological point of view. In : B. Leclercq, S. Richard, D. Seron (éds.), Objects and PseudoObjets : Ontological Deserts and Jungles from Brentano to Carnap. Boston Berlin : De Gruyter, p. 51-61.

[56] Brisart, R. (2016). La fin du correspondantisme. In : J.-F. Lavigne \& D. Pradelle (éds.), Monde, structures et objets de pensée : Recherches de phénoménologie en hommage à Jacques English. Paris : Hermann, p. 167-202.

[57] Brisart, R. \& Gauvry, C. (éds.) (2016). Perception et concept: Le conceptualisme en question. Bruxelles : Ousia.

[58] Crane, T. (2016). Le contenu non conceptuel de l'expérience, trad. R. Brisart. In [56], p. 185-216. [T. Crane (1992). The non-conceptual content of experience. In : T. Crane (éd.), The Contents of Experience: Essays on Perception. Cambridge: Cambridge University Press, p. 136-157.]

[59] Brisart, R. (2019\a). Textes posthumes I : Cours sur l'intentionnalité, éd. D. Seron. Bulletin d'analyse phénoménologique 15, 3, p. 1-34.

[60] Brisart, R. (2019 \b). Textes posthumes II : Philosophie continentale, de Nietzsche à Heidegger, éd. D. Seron. Bulletin d'analyse phénoménologique 15, 4, p. 1-90.

[61] Brisart, R. (2019 \c). Textes posthumes III : La contribution de Husserl à la no ready-made theory, éd. D. Seron. Bulletin d'analyse phénoménologique 15, 5, p. 1-29.

[62] Brisart, R. (2019\d). Textes posthumes IV : Sur la théorie de l'objet: Natorp versus Husserl, éd. D. Seron. Bulletin d'analyse phénoménologique 15, 6, p. 1-26.

[63] Brisart, R. (2019le). Textes posthumes V : À propos de l'argument de la Terre-jumelle, éd. D. Seron. Bulletin d'analyse phénoménologique 15, 7, p. 1-3.

[64] Brisart, R. (2019\f). Textes posthumes VI : Leçon sur Sartre et Husserl, éd. D. Seron. Bulletin d'analyse phénoménologique 15, 8, p. 1-10.

[65] Brisart, R. (à paraître). Le dépassement du correspondantisme : De Brentano à Husserl en passant par Kant. In: D. Pradelle \& P.J. Renaudie (éd.), Intentionnalité, sens, anti-psychologisme : Hommage à Robert Brisart. Hildesheim Zürich New York : Georg Olms. 